\title{
Follow-Up Consultation Through a Healthcare Kiosk for Patients with Stable Chronic Disease in a Primary Care Setting: A Prospective Study
}

\author{
Juliana Bahadin, MBBS, Mmed (Fam Med), FCFP, FAMS ${ }^{7}$, Eugene Shum, MBBS, MPH, FAMS ${ }^{2}$, \\ Grace Ng, BEng, MEng, Phd, MBBS, GDFM ${ }^{7,3}$, Nicolette Tan, BA ${ }^{2}$, Pushpavalli Sellayah, $B S C^{7}$, and \\ Sze Wee Tan, MBBS, MBA ${ }^{3}$
}

${ }^{7}$ SingHealth Polyclinics, Singapore, Singapore; ${ }^{2}$ Eastern Health Alliance, Centre for Innovation, Singapore, Singapore; ${ }^{3}$ Agency for Science, Technology and Research (A*STAR), Singapore, Singapore.

INTRODUCTION: The global healthcare kiosk market is growing, and kiosks are projected to be a larger part of healthcare delivery in the coming decades. We developed an unmanned healthcare kiosk that automates the management of stable patients with chronic conditions to complement face-to-face primary care physician (PCP) visits. AIM: The aim of our study was to show that the kiosk could be a feasible means of delivering care for stable patients with chronic conditions and could generate cost savings for the management of patients with stable chronic disease.

METHODS: We conducted a prospective single-arm study of 95 participants with well-controlled chronic cardiovascular diseases who visited our clinic in Singapore every 3 months for review and medication refill. During their subsequent appointments for chronic disease management at 3 and 6 months, participants used the kiosk instead of consulting a physician. All participants who used the kiosk were also evaluated by a nurse clinician (NC). The kiosk assessment of whether the patient was well controlled was then compared to the NC's assessment to determine rates of agreement. Patient satisfaction was evaluated through a questionnaire, and any adverse outcomes were documented.

RESULTS: Cohen's $\kappa$ for agreement between the kiosk and the NC assessment of patients' chronic care control was 0.575 (95\% CI, 0.437-0.713). The modest agreement was due to differences in systolic blood pressure measurements between the kiosk and the NC. The 96\% of participants who completed two kiosk visits were all satisfied with the kiosk as a care delivery alternative. None of the participants managed through the kiosk suffered any adverse outcomes. Use of the kiosk resulted in a reduction of 128 face-to-face PCP visits.

CONCLUSIONS: Healthcare kiosks can potentially be used to complement primary care clinician visits for managing patients with stable chronic diseases and can generate cost savings.

KEY WORDS: healthcare kiosk; stable chronic disease; alternative care delivery.

Received May 2, 2016

Revised September 14, 2016

Accepted November 14, 2016

Published online December 9, 2016
J Gen Intern Med 32(5):534-9

DOI: $10.1007 / \mathrm{s} 11606-016-3931-8$

(C) Society of General Internal Medicine 2016

\section{INTRODUCTION}

In Singapore, outpatient visits for the management of chronic diseases occur once every 3 months in government polyclinics, where almost half of patients with chronic diseases are treated. ${ }^{1,2}$ With the rising burden of chronic disease and an aging population, ${ }^{3}$ the demand for primary healthcare services has grown exponentially. At the same time, primary care physician (PCP) time is inefficiently consumed by routine, low-risk, non-urgent visits.

Various innovative models, including team-based care, ${ }^{4-6}$ minute clinics, ${ }^{7}$ and remote health services, ${ }^{8,9}$ are being implemented worldwide to aid in the care of patients. We developed the Self Empowering and Enabling Kiosk (SEEK), an unmanned healthcare kiosk, integrated with the electronic medical record, to complement office-based PCP visits. The global healthcare kiosk market is growing, and kiosks are projected to be a larger part of healthcare delivery in the coming decades. $^{10,11}$

This study is unique in assessing whether a healthcare kiosk can be used to complement PCP visits for delivering care to patients with well-controlled chronic disease, thus providing an alternative setting in which patients can receive care. Kiosks have the potential to increase access to primary healthcare services for new patients and to allow redistribution of resources for the management of more complex patients who require greater time and more intensive care. If effective, kiosks have the potential to automate, to some extent, the delivery of healthcare in the same way that ATMs do for banking.

Our aim in this work was to evaluate the SEEK kiosk as a care delivery tool and to determine its feasibility as an alternative means of delivering care for patients with stable chronic disease. We hypothesized that there would be at least moderate agreement in the assessment of chronic disease control between the kiosk and a nurse clinician (NC), that participants would be satisfied with the kiosk, and that participants who consulted the 
kiosk would not have adverse outcomes. Our secondary aim was to show that the kiosk could generate potential cost savings for the management of stable chronic disease in primary care.

\section{METHODS}

\section{Study Design and Population}

We conducted a prospective study and enrolled a convenience sample of 100 participants. Patients who were included were 18 to 70 years old, with at least one of the following chronic diseases: hypertension, hyperlipidemia, or diabetes mellitus, with blood pressure (BP), low-density lipoprotein cholesterol (LDL-C), and hemoglobin A1c (HbAlc) values at target. We excluded patients with levels of creatinine $>140 \mathrm{mmol} / \mathrm{l}$, potassium $>3.5 \mathrm{mmol} / 1$ or $>5 \mathrm{mmol} / 1$, newly started on angiotensin-converting-enzyme (ACE) inhibitor or angiotensin receptor blocker, recent discharge from hospital or specialist outpatient clinic, need for laboratory exams except for HbAlc, or any new complaints related to the management of chronic disease.

Kiosk Deployment. Before launching the kiosk, we conducted rigorous tests and simulations. The kiosk was subjected to numerous simulated participant scenarios as well as a dry run with mock participants. However, for the first 17 subjects, we found inaccurate kiosk decisions for wellcontrolled, sub-optimally controlled, and poorly controlled patients. As a result, these participants were given advice based on the wrong classification by the kiosk, thus resulting in inappropriate disease management. ${ }^{12}$ We corrected the algorithm, and we excluded this data from the current analysis. Of these 17 participants, 12 participants were evaluated by the $\mathrm{NC}$ and found to be well controlled, and thus allowed to continue with kiosk visit 2 . Therefore, only 95 participants ultimately continued with the study after the kiosk accuracy issue was resolved.

Kiosk Decision Algorithm. The kiosk was designed to classify participants into three groups: those with wellcontrolled, sub-optimally controlled, or poorly controlled disease (Table 1). The algorithm used by the kiosk was generated using patients' cardiovascular risk according to coronary artery disease risk factors, ${ }^{13}$ responses to the participant questionnaire, BP and pulse measurements, and laboratory values. All participants were asked, through a questionnaire built into the kiosk, whether during the past month they had 1) experienced chest pain, 2) experienced breathlessness, or 3) been admitted to a hospital. If the participant answered "yes" to any of the questions, the kiosk classified them as having red flag symptoms, and they were directed to see a physician and were excluded from the study.

Kiosk Visits. Figure 1 shows the flow of participant visits. The first and second kiosk visits occurred 3 and 6 months after recruitment. After the kiosk provided a determination of whether the patient's condition was well controlled, an NC independently evaluated and classified the participant using parameters identical to those of the kiosk. If the $\mathrm{NC}$, who was blinded to the kiosk finding, found that the participant's disease was sub-optimally or poorly controlled, the participant was referred to the primary care physician for further management and excluded from the study. At the end of the second kiosk visit, participants were required to answer a questionnaire built into the kiosk to evaluate their satisfaction with the use of the kiosk as an alternative patient care method.

At 9 months, all participants who continued with the study were reviewed by a PCP. The PCP evaluated any adverse outcomes as a result of not consulting a PCP at the 3- and 6month intervals.

\section{PRIMARY OUTCOME}

The primary outcome was agreement between the $\mathrm{NC}$ and the kiosk regarding the classification of subjects as well controlled, sub-optimally controlled, or poorly controlled according to their cardiovascular risk factors.

\section{SATISFACTION}

We used four satisfaction questions, with responses based on a four-point Likert scale from strongly disagree to strongly agree. The survey questions related to the

Table 1 Kiosk Decision and Management Algorithm

\begin{tabular}{|c|c|c|c|}
\hline $\begin{array}{l}\text { Positive ("yes") answer to any } \\
\text { questionnaire question }\end{array}$ & No & No & Yes \\
\hline Blood pressure (mmHg) & $\begin{array}{l}90 / 60 \text { to the maximum } \\
\text { allowed target } \mathrm{BP}\end{array}$ & $\begin{array}{l}\text { Above maximum allowed target BP to } \\
159 / 99\end{array}$ & $\geq 160 / 100$ or below target \\
\hline LDL-C (mmol/l) & Below target & $\begin{array}{l}\text { Above maximum allowed target to below } \\
\text { target set for next risk category }\end{array}$ & $\begin{array}{l}\text { Above target set for next risk } \\
\text { category, or } 4.9 \mathrm{mmol} / 1\end{array}$ \\
\hline HbAlc (\%) & $6.0-7.0$ & $7.1-8.0$ & $>8.0$ or below target \\
\hline Pulse rate (beats/min) & $50-105$ & $>105$ & Below target \\
\hline $\begin{array}{l}\text { Kiosk classification if any of the } \\
\text { above criteria are met }\end{array}$ & Well controlled & Sub-optimally controlled & Poorly controlled \\
\hline
\end{tabular}




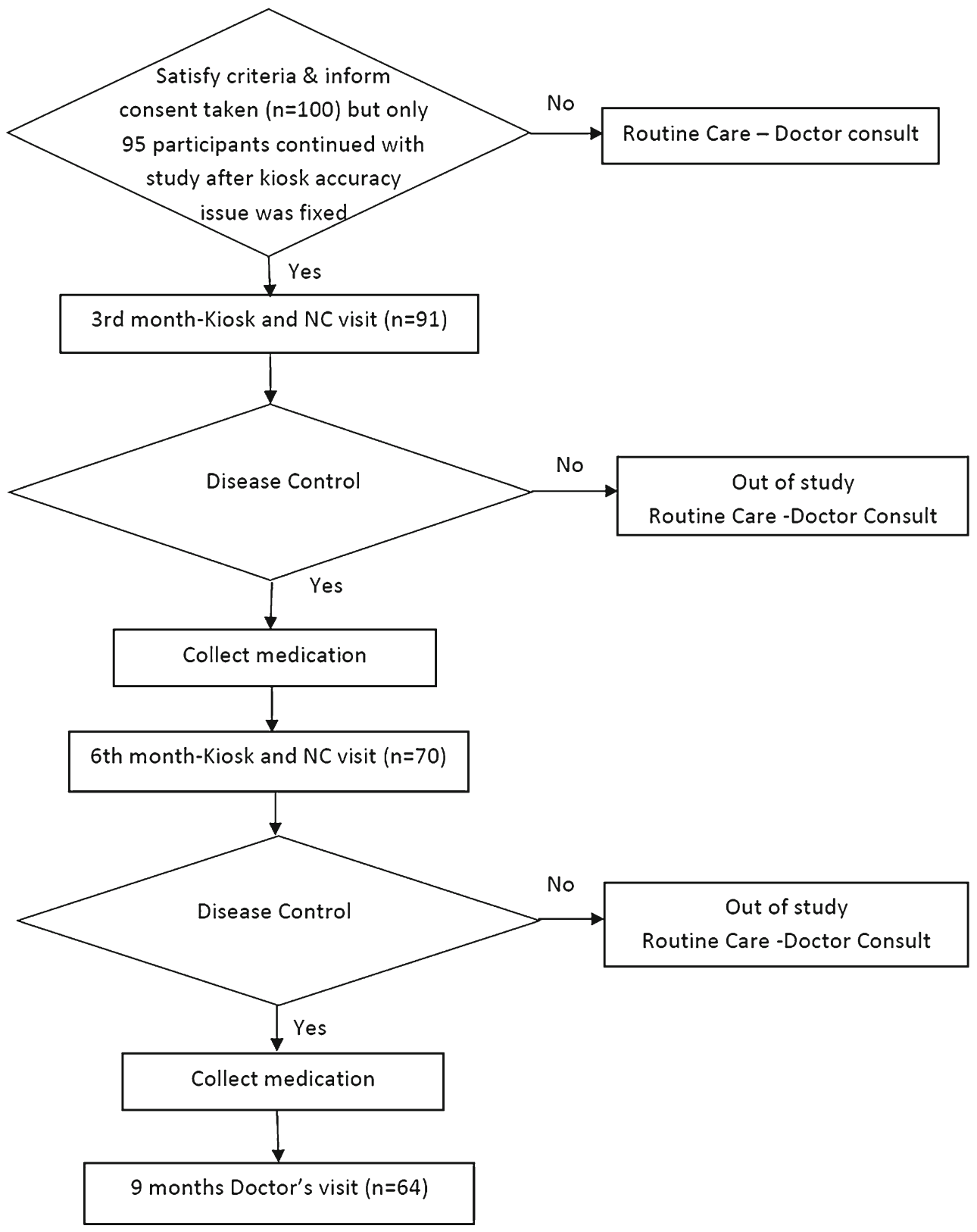

Figure 1 Flow chart of patient participation in the study.

kiosk's ease of use, ease of instructions, ability to replace the provider, and amount of time spent using the kiosk. We considered a participant satisfied if he or she agreed or strongly agreed with all four satisfaction questions.

\section{BLOOD PRESSURE MEASUREMENTS}

The kiosk uses an Omron HBP-9020 BP measuring device (Omron Healthcare, Kyoto, Japan), which has been approved by the Health Sciences Authority of Singapore and meets standards in accordance with the Health Products Act and Health Products (Medical Devices) Regulations. The NC used the GE Carescape V100 vital signs monitor (GE Healthcare, Chicago, IL, USA) for BP measurement.

\section{EVALUATION OF COSTS}

Our facility is subsidized by the government, and the unit cost per physician visit was $\$ 41.68$. This cost does not include medication or laboratory charges. A large proportion of the cost is provided by the government, while the actual cost to patients is based on the patient age group. Therefore, cost savings from the kiosk were estimated by multiplying the reduction in the number of physician visits by the unit cost per physician visit.

\section{STATISTICAL ANALYSIS}

We used proportions and means to describe the study sample. We used the paired $t$ test to assess differences 
between the BP measurements by the kiosk and the NC, and Cohen's kappa to assess agreement between the kiosk decision and the NC's decision. All statistical tests were two-tailed, and a $p$ value of $<0.05$ was considered statistically significant.

\section{RESULTS}

\section{Baseline Characteristics}

Table 2 presents the baseline characteristics of the sample. Our study started with 95 participants; 91 participants completed one visit, 70 participants completed two visits, and 64 participants completed the 9-month visit. Seven participants withdrew from the study for personal reasons, and 29 exited the study because their chronic condition was not well controlled and required a consultation with a doctor. The reasons given for withdrawing and exiting from the study are shown in Table 3. The most common reason for withdrawal was a preference for seeing a physician, and the most common reasons for exiting the study were uncontrolled $\mathrm{BP}$ or $\mathrm{HbA} 1 \mathrm{c}$ outside the target range.

\section{Agreement Between the Nurse Clinician and Kiosk Assessment}

At the first visit, $124(83 \%)$ of the kiosk assessments matched the NC's assessment. Cohen's $\mathrm{k}$ was 0.575 (95\% CI, 0.437-0.713) for categorizing the participants into well-controlled, sub-optimally controlled, and poorly controlled groups.

The main reason for the modest agreement was a difference in BP measurements between the kiosk and the NC. The mean systolic BP measured by the kiosk was $132.7 \mathrm{mmHg}( \pm 12.7 \mathrm{mmHg})$, and the mean systolic BP measured by the $\mathrm{NC}$ was $125.5 \mathrm{mmHg}( \pm 10.4 \mathrm{mmHg}$; $p<0.01)$ The mean diastolic BP measured by the kiosk was $76.7 \mathrm{mmHg}( \pm 8.2 \mathrm{mmHg})$, while the mean diastolic

Table 2 Participant Characteristics

\begin{tabular}{ll}
\hline \hline Characteristic & \\
\hline Number of participants & 100 \\
Mean age, years (standard deviation) & $61.4( \pm 6.7)$ \\
Women (\%) & 55 \\
Race/ethnicity (\%) & 74 \\
Chinese & 21 \\
Malay & 3 \\
Indian & 2 \\
Other & 1 \\
Education level (\%) & 20 \\
None & 35 \\
Primary & 14 \\
Secondary & 84 \\
Tertiary & 23 \\
Hypertension (\%) & 89 \\
Diabetes (\%) & \\
Hyperlipidemia (\%) & \\
\hline
\end{tabular}

Table 3 Reasons for Participant Withdrawal/Exit from the Study

\begin{tabular}{|c|c|c|}
\hline \multicolumn{2}{|l|}{ Reason for withdrawal } & $\begin{array}{l}\text { Number of } \\
\text { participants }(n=7)\end{array}$ \\
\hline \multicolumn{2}{|l|}{ Preferred to see a doctor } & 2 \\
\hline \multicolumn{2}{|c|}{ Disallowed by family to participate } & 1 \\
\hline \multicolumn{2}{|c|}{$\begin{array}{l}\text { Needed certification from doctor for time off } \\
\text { during review }\end{array}$} & 1 \\
\hline \multicolumn{2}{|c|}{ Posted for overseas work assignment } & 1 \\
\hline \multicolumn{2}{|c|}{$\begin{array}{l}\text { Wanted 6-month medication supply at one } \\
\text { time }\end{array}$} & 1 \\
\hline \multicolumn{2}{|c|}{ Came for unscheduled doctor visit } & 1 \\
\hline Reason for exit & $\begin{array}{l}\text { No. of patients } \\
\text { exited at } 3 \text { months } \\
(n=19)\end{array}$ & $\begin{array}{l}\text { No. of patients exited } \\
\text { at } 6 \text { months }(n=5)\end{array}$ \\
\hline HbA1c below target & 3 & 1 \\
\hline $\begin{array}{l}\text { HbA1c below target } \\
\text { and BP above target }\end{array}$ & 1 & \\
\hline HbA1c above target & 3 & \\
\hline BP above target & 7 & 2 \\
\hline LDL above target & 1 & \\
\hline $\begin{array}{l}\mathrm{BP} \text { and } \mathrm{HbA} 1 \mathrm{c} \text { above } \\
\text { target }\end{array}$ & 2 & \\
\hline BP below target & 1 & \\
\hline $\begin{array}{l}\text { With red flag } \\
\text { symptoms }\end{array}$ & & 1 \\
\hline $\begin{array}{l}\text { With red flag } \\
\text { symptoms and BP } \\
\text { above target }\end{array}$ & 1 & 1 \\
\hline
\end{tabular}

BP measured by the $\mathrm{NC}$ was $73.0 \mathrm{mmHg}( \pm 7.9 \mathrm{mmHg}$; $p<0.05)$. The same trend was seen at visit 2 .

\section{Patient Satisfaction with Kiosk}

Seventy patients who completed the second kiosk visit completed the patient satisfaction survey. Table 4 shows the results of the patient survey. All of the participants agreed or strongly agreed that the kiosk was easy to use, and $96 \%$ agreed or strongly agreed that they could use the kiosk instead of a physician.

\section{Adverse Events}

As part of the study protocol, all study participants were required to have a doctor visit at 9 months. These 64 patients had not had any hospitalizations or additional doctor consultations, nor did the doctor identify any red flag symptoms that had not been detected by the kiosk. All but two patients met the targets for BP, LDL, and $\mathrm{HbAlc}$ at 9 months. One of the participants who did not meet his BP target at the doctor's visit had BP above target at visit 1 as assessed by the kiosk, but when rechecked by the $\mathrm{NC}$, his BP was lower and met the target. The other participant who did not meet the target BP at 9 months had BP that was only slightly above target, at $141 / 78 \mathrm{mmHg}$.

\section{Potential Cost Savings}

Sixty-four percent of patients with well-controlled chronic disease were able to replace two PCP visits with kiosk visits, with no adverse outcome. This constitutes a reduction of 128 physician visits, which translates to total savings of $\$ 5335$. 
Table 4 Patient Satisfaction Survey

\begin{tabular}{|c|c|c|c|c|}
\hline Question & 1 = Strongly disagree & $2=$ Disagree & $3=$ Agree & $4=$ Strongly agree \\
\hline The instructions for using the kiosk were easy to understand. & $0 \%$ & $4 \%$ & $60 \%$ & $36 \%$ \\
\hline The equipment in the kiosk was easy to use. & $0 \%$ & $0 \%$ & $69 \%$ & $31 \%$ \\
\hline The time spent at the kiosk was just right. & $0 \%$ & $0 \%$ & $69 \%$ & $31 \%$ \\
\hline I can use the kiosk instead of seeing the doctor. & $0 \%$ & $4 \%$ & $63 \%$ & $33 \%$ \\
\hline
\end{tabular}

\section{DISCUSSION}

Our study showed moderate agreement between the SEEK kiosk and evaluation by a nurse clinician. The main discordance between the two was that the kiosk BP reading was higher than that of the $\mathrm{NC}$, resulting in participants being categorized as having suboptimal or poor control by the kiosk versus well-controlled or sup-optimally controlled status by the NC.

This difference in BP readings may be due to the fact that BP measurements change from minute to minute and can be affected by various factors. ${ }^{14}$ The kiosk uses the Omron HBP9020 BP measuring device, which has been approved by the Health Sciences Authority of Singapore and meets the standards in accordance with the Health Products Act and Health Products (Medical Devices) Regulations. We conducted a quality evaluation of the device and found that the readings were accurate.

We relied on single BP measurements by the kiosk and $\mathrm{NC}$ instead of repeated measurements. Unfortunately, single BP measurements do not take into account beat-to-beat variability and at best are a rough estimate of the patient's actual average $\mathrm{BP},{ }^{15}$ which may also account for the observed differences.

Another potential factor to account for the difference is the patient's level of anxiety when using the kiosk. Patient emotions also affect variability in BP. ${ }^{16-18}$ This theory is supported by the greater difference in mean systolic BP at visit 1 than visit 2 , which could be as a result of the exposure to a new measurement protocol in contrast to the familiar face of the NC. Thus, when patients returned to the kiosk for the second visit, they were already familiar with the kiosk, and so the mean difference in systolic BP between kiosk and $\mathrm{NC}$ was lower.

The main limitation of this study is its lack of a control arm. However, the aim of the study was to evaluate the feasibility and acceptability of using the kiosk to provide care. A randomized design with a control group would not be able to evaluate agreement between clinician and kiosk, and could only provide results regarding satisfaction with and appropriateness of treatment. Future research is needed to compare the health outcomes of well-controlled patients with chronic conditions using the kiosk to those seeing physicians.

The study also showed that all participants who completed two kiosk visits found the kiosk easy to use and felt that the kiosk was a viable alternative to seeing a doctor. This is an important finding, since the majority of our sample was elderly, and this population was able to adopt an innovative model of care that leveraged technology. A 2009 study by Luo et al. ${ }^{19}$ in a representative sample of 705 patients visiting a polyclinic in Singapore (mean age 54.6 years) showed that only $40 \%$ of the patients were comfortable using the internet or texting technology for healthcare delivery. The majority of patients preferred face-to-face doctor visits, thereby creating a barrier to the implementation of new technology to support delivery of care.

In our clinics, $30 \%$ of our cardiovascular patients have well-controlled disease, and thus would be eligible for kiosk consultation. We estimate that 8200 well-controlled patients could have two face-to-face PCP visits per year replaced by the kiosk, which would be a savings of 16,400 doctor visits. This would translate to an annual savings of approximately $\$ 683,552$. The approximate commercial cost of the kiosk is $\$ 10,000$ per year, and the annual operating cost is $\$ 4000$. Thus, the total cost of a kiosk is $\$ 14,000$, and if two kiosk are used, the total kiosk expenses are $\$ 28,000$. As such, the cost of delivering care to a stable chronic patient using a kiosk is about $4 \%$ of the current cost of delivering care by face-to-face PCP visits. Even if one in five eligible patients with well-controlled chronic disease were to use the kiosk, the cost of delivering care would be only about $20 \%$ of the cost of face-to-face care.

Another potential advantage of the kiosk is the time savings for the patient. For the purpose of this study, the kiosk was placed in the clinic waiting area. In the future, the kiosk will be placed outside the clinic environment in a place accessible to patients at any time of day, and not limited to clinic hours. Patients with stable chronic disease currently wait half a day to see a PCP for management of their condition. When the kiosk is used as a care delivery alternative, the patient needs to spend only about $7 \mathrm{~min}$ at the kiosk, and this can be done outside working hours. The reduction of two doctor visits equates to a savings of one work day for the patient or their employers every 6 months. Based on mean salaries ${ }^{20}$ of Singaporeans, this represents a savings of $\$ 173.50$ for the patient.

Healthcare kiosks should not be used in isolation, as a replacement for healthcare providers. Different patients may have different preferences, and preferences may change over time. As healthcare providers, we should strive to provide a variety of care options to meet the needs of our patients. With advances in remote and mobile health technology, virtual consultation, ${ }^{21}$ synchronous and asynchronous telehealth modalities, ${ }^{22,23}$ and email consultations, ${ }^{24,25}$ we can now offer multiple avenues of care. These, together with the healthcare kiosk, can be used by patients with chronic diseases to 
improve quality of care, patient empowerment, and access to care. Only by increasing the variety of care delivery modalities can we then reach out to more of the patient population, and begin to cope with the global burden of chronic disease. ${ }^{26}$

While our study showed that a kiosk can be a feasible care delivery option, with good patient acceptance, this was a pilot study involving a small number of patients. A more robust, controlled study is needed to fully evaluate whether the kiosk can indeed serve as an alternative care delivery option with a potential cost benefit.

Acknowledgements: The authors thank Dr. Tan Ngiap Chuan, Ms. Patricia Kin, Ms. Caris Thong, and Ms. Eileen Koh from the Department of Research, SingHealth Polyclinic, for their invaluable help and support. We would also like to thank our research coordinators Ms. Susan Toh and Ms. Cheah Seng Lian, and our nurse clinicians SSN Lee Yuet Lai, SSN Woo Yuen Kau, SSN Teo Sok Hoon, and SSN Munahwarah Bte Mokhtar for their assistance. Our special thanks also to Ms. Claudia Chow, Ms. Lydia Tan, and Mr. TK Udairam from the Eastern Health Alliance Centre for Innovation for making this project possible. The kiosk was designed and manufactured by AIT Technologies Pte Ltd. This work was funded by an A*STAR-CIMIT [Center for Integration of Medicine and Innovative Technology] Commercialization Project grant.

Corresponding Author: Juliana Bahadin, MBBS, Mmed (Fam Med), FCFP, FAMS; SingHealth Polyclinics, 167 Jalan Bukit Merah, Tower 5, \#15-10, Singapore 150167, Singapore (e-mail: juliana. bahadin@singhealth.com.sg).

\section{Compliance with Ethical Standards:}

Conflict of Interest: The authors declare that they do not have a conflict of interest.

\section{REFERENCES}

1. Singapore Health Facts 2014 Ministry of Health Singapore. Available at:https://www.moh.gov.sg/content/moh_web/home/statistics/Health Facts_Singapore/Top_4_Conditions_of_Polyclinic_Attendances.html. Accessed October 26, 2016

2. Primary Care Survey 2010. Profile of primary care patients by Song Qing Si Ministry of Health Singapore. Available at: https://www.moh.gov.sg/content/dam/moh_web/Publications/Information\%20Papers/2011/Primary\%20Care\%20Survey $\% 202010 \% 20$-20Profile\%20of\%20Primary\%20Care\%20Patients.pdf. Accessed October 26, 2016

3. National Health Survey 2010 Ministry of Health Singapore. Available at: https://www.moh.gov.sg/content/dam/moh_web/Publications / Reports/2011/NHS2010\%20-\%20low\%20res.pdf. Accessed October 26, 2016

4. Jesmin S, Thind A, Sarma S. Does team-based primary health care improve patients' perception of outcomes? Evidence from the 2007-08 Canadian Survey of Experiences with Primary Health. Health Policy. 2012;105(1):71-83

5. Taylor E, Rachel M, Meyers D, et al. Enhancing the primary care team to provide redesigned care: The roles of practice facilitators and care managers. Ann Fam Med. 2013;11(1):80-83.

6. Borrill C, West $\mathbf{M}$, Shapiro $\mathbf{D}$, et al. Team working and effectiveness in health care. Br J Healthc Manag. 2000;6(8):364-371.
7. Hoffmann A. Minute medicine: examining retail clinic legal issue and legislative challenges health matrix. J Law Med. 2010;20(2):467-497.

8. Rosati R. Evaluation of remote monitoring in home health care. 2009 International Conference on eHealth, Telemedicine, and Social Medicine 2009:25-27

9. Bayer S, Barlow J, Curry R. Assessing the impact of a care innovation: telecare. Syst Dyn Rev. 2007;23(1):61-80.

10. Self-service technology doctors up health care, pt. 1. Available at: http:// www.kioskmarketplace.com/articles/self-service-technology-doctors-uphealth-care-pt-i/. Accessed October 26, 2016

11. A New Age for Healthcare Kiosks-Five Ways Next Generation Kiosks Disrupt Medicine and Healthcare Marketing. Available at: http://www. forbes.com/sites/stephenwunker/2013/07/16/a-new-age-for-healthcare-kiosks-five-ways-next-generation-kiosks-disrupt-medicine-andhealthcare-marketing/\#5e4e50396d7. Accessed October 26, 2016

12. Ng G, Tan N, Bahadin J, et al. Development of an automated healthcare kiosk for the management of chronic disease patients in the primary care setting. J Med Syst. 2016;40(7):1-11. doi:10.1007/s10916-016-0529-y.

13. Ministry of Health Singapore Clinical Practice Guidelines 1) Diabetes July 2006 2) Lipids May 2006 3) Hypertension June 2005. Available at: https://www.moh.gov.sg/content/moh_web/healthprofessionalsportal/ doctors/guidelines/cpg_medical_withdrawn.html. Accessed October 26. 2016

14. O'Brien E, Asmar R, Beilin L, et al. European Society of Hypertension Working Group on Blood Pressure Monitoring. European Society of Hypertension recommendations for conventional, ambulatory and home blood pressure measurement. J Hypertens. 2003;21:821-848.

15. Pickering TG, Hall JE, Appel LJ, et al. Recommendations for blood pressure measurement in humans and experimental animals: Part 1: blood pressure measurement in humans: a statement for professionals from the Subcommittee of Professional and Public Education of the American Heart Association Council on High Blood Pressure Research. Hypertension. 2005;45:142-161.

16. Mancia G, Parati G, Casadei R, et al. Effect of stress on diagnosis of hypertension. Hypertension. 1991;17(supplIII):III-56-III-62.

17. Mancia G, Bertinieri G, Grassi G, et al. Effects of blood pressure measurements by the doctor on patient's blood pressure and heart rate. Lancet. 1983;2:695-698.

18. Mancia G, Parati G. Reactivity to physical and behavioural stress and blood pressure variability in hypertension. In: Julius S, Bassett DR, eds. Handbook of hypertension: volume 9, behavioural factors in hypertension. Amsterdam: Elsevier; 1987:104-122.

19. Luo N, Koh WP, Ng WY, et al. Acceptance of information and communication technologies for healthcare delivery: a SingHealth Polyclinics study. Ann Acad Med Singapore. 2009;38(6):529-8.

20. Gross monthly income from works. Available at: http://stats.mom.gov. sg/Pages/Income-Summary-Table.aspx. Accessed October 26, 2016.

21. Dixon RF, Stahl JE. A randomized trial of virtual visits in a general medicine practice. J Telemed Telecare. 2008;14(2):62-6. doi:10.1258/jtt. 2007.070709.

22. Dellifraine $\mathbf{J L}$, Dansky KH. Home-based telehealth: a review and metaanalysis. J Telemed Telecare. 2009; 15(3):115-7. doi:10.1258/jtt.2009.003003.

23. Huang $\mathbf{Z}$, Tao $\mathbf{H}$, Meng $\mathbf{Q}$, et al. Management of endocrine disease. Effects of telecare intervention on glycemic control in type 2 diabetes: a systematic review and meta-analysis of randomized controlled trials. Eur J Endocrinol. 2015;172(3):R93-101. doi:10.1530/EJE-14-0441.

24. Neville RG, Marsden W, McCowan C, et al. Email consultations in general practice. Inform Prim Care. 2004;12(4):207-14.

25. Car J, Sheikh A. Email consultations in health care: 1-scope and effectiveness. BMJ. 2004;329(7463):435-438.

26. Yach D, Hawkes C, Linn Gould C, et al. The global burden of chronic diseases: overcoming impediments to prevention and control. JAMA. 2004;291(21):2616-2622. doi:10.1001/jama.291.21.2616. 